\title{
Developmental Changes Underlying the Formation of the Specialized Time Coding Circuits in Barn Owls (Tyto alba)
}

\author{
M. Fabiana Kubke, Dino P. Massoglia, and Catherine E. Carr \\ Department of Biology, University of Maryland, College Park, Maryland 20742
}

Barn owls are capable of great accuracy in detecting the interaural time differences (ITDs) that underlie azimuthal sound localization. They compute ITDs in a circuit in nucleus laminaris (NL) that is reorganized with respect to birds like the chicken. The events that lead to the reorganization of the barn owl NL take place during embryonic development, shortly after the cochlear and laminaris nuclei have differentiated morphologically. At first the developing owl's auditory brainstem exhibits morphology reminiscent of that of the developing chicken.

The barn owl's (hereafter referred as owl) auditory system uses interaural time differences (ITDs) and interaural level differences (ILDs) for sound localization. Coding of ITDs relies on coincidence detection by nucleus laminaris (NL) neurons, which are sensitive to binaural ongoing phase disparities (Moiseff and Konishi, 1983). The circuit responsible for the computation of ITDs in birds has been studied extensively in chickens and owls (Ramón y Cajal, 1908; Smith and Rubel, 1979; Young and Rubel, 1983; Carr and Konishi, 1988; Carr and Konishi, 1990; Overholt et al., 1992; Carr and Boudreau, 1993; Joseph and Hyson, 1993). The auditory nerve conveys phase-locked information to the cochlear nucleus magnocellularis (NM), which in turn projects bilaterally to NL. Ipsilateral and contralateral inputs from NM to NL conform to the Jeffress model for sound localization (see Fig. 1) (Jeffress, 1948), creating a coherent map of azimuthal space.

Each element of this circuit must be precisely matched to faithfully transform temporal information into a coherent map. Thus the morphological characteristics of the circuit are needed to understand its operation. Three principal organizational modifications differentiate the ITD-coding circuits of chickens and owls: the organization of the cell bodies within NL, the distribution of dendrites within each NL neuron, and the organization of the inputs from NM to NL. In the chicken, NL consists of a single layer of cells in a laminar structure extending along the rostromedial to caudolateral axis (see Fig. $1 A$ ). In contrast, owl NL neurons have their cell bodies interspersed throughout a 1-mmthick neuropil with no apparent laminar organization (see Fig.

\footnotetext{
Received Dec. 19, 2001; revised May 14, 2002; accepted May 14, 2002.

This work was supported by National Institutes of Health Grant DCD000436 to C.E.C. We thank Dr. K. Buckley for her generous gift of the SV2 antibody. We gratefully acknowledge L. Rigby, T. Maugel, A. Rzasa, and J. Entlich for technical assistance, Drs. A. Cohen, L. Katz, and R. Mooney for the use of digital equipment, and a reviewer for providing the Matlab script used for the dip test.

Correspondence should be addressed to C. E. Carr, Department of Biology, University of Maryland, College Park, MD 20742. E-mail: cc117@umail.umd.edu.

M. F. Kubke's present address: Division of Anatomy, Faculty of Medical and Health Sciences, University of Auckland, Box 92019, Auckland, 1001 New Zealand.

D. P. Massoglia's present address: Wake Forest University School of Medicine, Department of Neurobiology and Anatomy, Medical Center Boulevard, WinstonSalem, NC 27157.

Copyright (C) 2002 Society for Neuroscience $\quad 0270-6474 / 02 / 227671-09 \$ 15.00 / 0$
}

Later, the two systems diverge, and the owl's brainstem auditory nuclei undergo a secondary morphogenetic phase during which NL dendrites retract, the laminar organization is lost, and synapses are redistributed. These events lead to the restructuring of the ITD coding circuit and the consequent reorganization of the hindbrain map of ITDs and azimuthal space.

Key words: avian development; morphogenesis; auditory; laminaris; evolution; interaural time difference

$1 C)$. Neurons in the chicken are bitufted, projecting their dendrites toward the dorsal and ventral surfaces of the nucleus (Ramón y Cajal, 1908; Smith and Rubel, 1979; Smith, 1981; Jhaveri and Morest, 1982). Owl neurons exhibit short dendrites distributed throughout their cell bodies with no evident polarization (Carr and Boudreau, 1991, 1993). Ipsilateral and contralateral NM inputs to NL in the chicken are segregated, such that dorsal dendrites receive exclusively ipsilateral NM projections, whereas ventral dendrites are contacted exclusively by contralateral NM axons (Young and Rubel, 1983). This segregation is absent in owls. Instead, NM axons enter NL and traverse it, making synaptic contacts on NL neurons along the entire dorsoventral dimension (Carr and Boudreau, 1991). This morphological restructuring results in a reorganization of the delay lines and the associated map of ITDs within NL. In the chicken, position along the azimuth is mapped along the mediolateral axis (Overholt et al., 1992), whereas in the owl multiple maps of ITD emerge along the dorsoventral axis (Sullivan and Konishi, 1986).

Because these anatomical variations may contribute to the increased acuity of sound localization seen in owls, we sought to identify the developmental process that lead to the reorganization of the NM-NL circuit in owls. During embryogenesis, the owl brainstem exhibits an organization resembling that described in chicken. Later, the owl's auditory nuclei undergo a second morphogenetic phase that leads to the restructuring of the time coding circuit.

\section{MATERIALS AND METHODS}

Data are based on results from 58 owl embryos (Tyto alba) ranging from embryonic day (E) 8 to hatching (incubation period of 32 d). Owl embryos were staged according to Meyer and Wagner (1995). Other material from these animals has been used in parallel studies (Massoglia, 1997; Kubke and Carr, 1998; Kubke et al., 1999; Cheng, 2001). All animal experimental procedures were approved by the University of Maryland Animal Care and Use Committee and followed National Institutes of Health guidelines.

\section{Light microscopy studies}

Nissl material. Fifteen owl embryos (E8-E31) were processed for paraffin sections. Owl eggs were incubated at $37^{\circ} \mathrm{C}$ in a forced draft humidified 
Figure 1. Schematic showing the Jeffress model for sound localization as applied to chicken and owl. The Jeffress model proposed that time differences can be measured with the use of two elements: delay lines and coincidence detectors. A coincidence detector will fire maximally when the input from both sides arrives at the same time, that is, when the delay of the onset of the stimuli is equal and opposite to the retardation imposed by the delay line. $A$, Schematic cross section through a chicken brainstem showing the organization of the projections from NM to NL. This organization conforms to a modified Jeffress model $(B)$ with delay lines formed by the contralateral NM axons that run ventral to NL. This results in a map of ITD oriented in the mediolateral dimension, with cells in more lateral positions responding maximally to sounds originating from far contralateral space, and cells in a more medial position responding maximally to sounds originating from the front. $C$, Schematic cross section through an owl brainstem showing the organization of the projections from NM to NL. The organization of the delay lines conforms to the Jeffress model
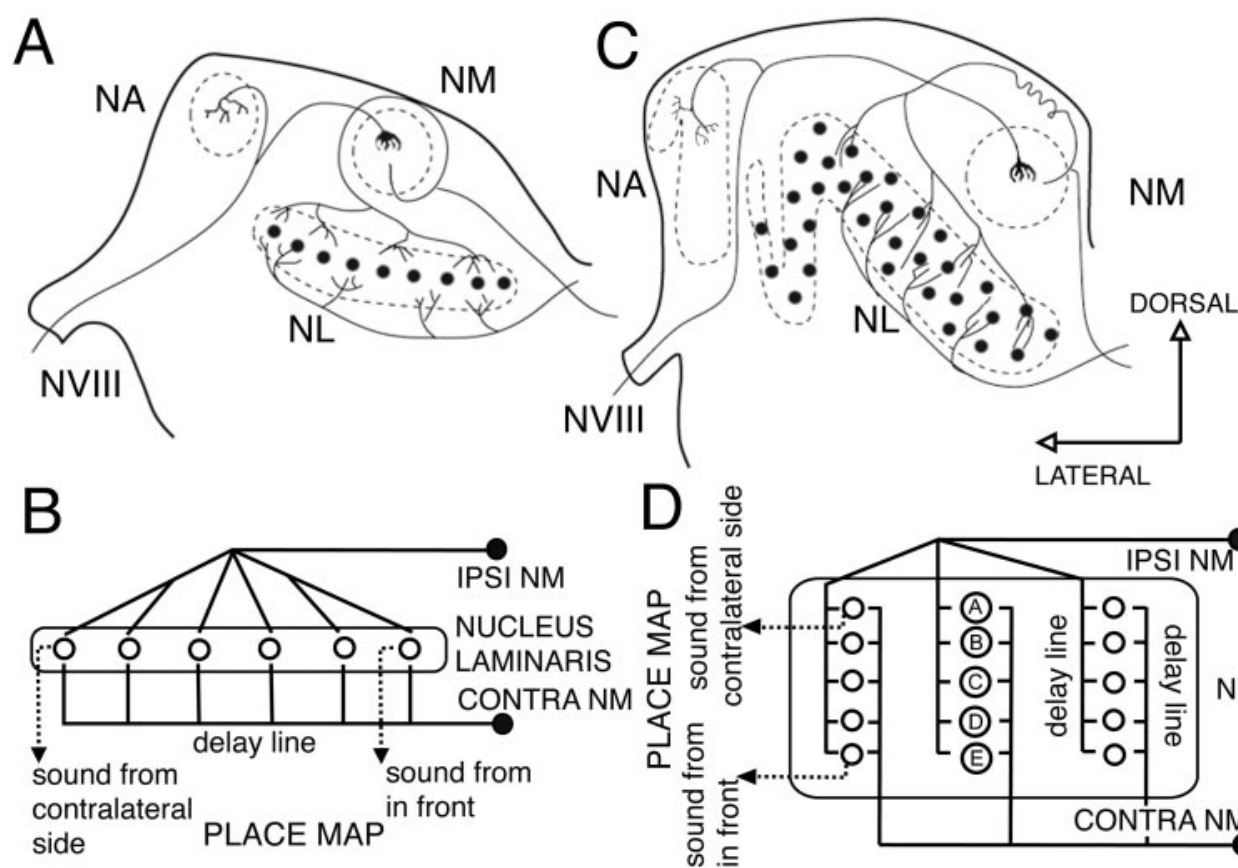
$(D)$. NM axons enter NL and traverse it, making contact with NL neurons $(A-E)$ along their way. This results in multiple maps of ITD $(D)$ with space mapped in a dorsoventral dimension. Neurons located in the dorsal edge of NL respond maximally to sounds originating from far contralateral space (neuron $A$ ), and neurons located in more ventral positions respond maximally to sounds originating from the front (neuron $E$ ). (Modified from Kubke and Carr, 2000). VIII, Cochleovestibular nerve; $N A$, nucleus angularis; $N M$, nucleus magnocellularis; $N L$, nucleus laminaris.

environment with regular turning. Embryos were killed by decapitation and fixed in AFA (70\% EtOH/Formalin/Glacial acetic acid). After a minimum of $3 \mathrm{~d}$ in fixative, the tissue was dehydrated in an ethanol series followed by t-butyl alcohol. The tissue was embedded in paraffin and sectioned at $10 \mu \mathrm{m}$ in the coronal axis. Sections were stained with cresyl violet. Line drawings were obtained by digital tracing of cresyl violetstained material. Digital images were captured using a Magnafire camera attached to a Nikon microscope. Section outlines were traced using Corel Draw 9, and dots were placed on top of each cell nucleus.

Golgi impregnation. The brains of 13 embryos (E13-E31) were impregnated by Golgi methods. Two brains were prepared according to the rapid Golgi technique (Valverde, 1970), and the rest were prepared according to the method of Stensaas (1967). The brains were removed from the skull (E26 and older), or whole heads were trimmed and postfixed in mordant for 2-4 d. The brains were rinsed in water and transferred to silver nitrate for 1-3 d. They were embedded in celloidin and cut in the coronal plane at 120 or $150 \mu \mathrm{m}$. Impregnated neurons were drawn under $1000 \times$ magnification, using a camera lucida attached to an Olympus microscope.

Immunohistochemistry. Twenty-five owl embryos (E11-hatching) were processed for immunocytochemistry. Owls were anesthetized with ketamine (15 mg/kg, i.m.) followed by an overdose of pentobarbital (35 $\mathrm{mg} / \mathrm{kg}$, i.m.). After intracardiac injection of heparin, owls were perfused with saline, followed by $4 \%$ paraformaldehyde (PF) in $0.1 \mathrm{M}$ phosphate buffer (PB, pH 7.4). The brains were postfixed in the perfusion solution for $1-2 \mathrm{~d}$ and cryoprotected in $30 \%$ sucrose in $\mathrm{PB}$ at $4^{\circ} \mathrm{C}$. Brains were embedded in $7.5 \%$ gelatin $/ 30 \%$ sucrose $/ 0.1 \mathrm{M}$ PB and placed in $30 \%$ sucrose $/ 4 \%$ PF/0.1 M PB overnight. Good histological preservation was achieved without a marked loss of antigenicity using these protocols, or after prolonged periods of postfixation. The brains were sectioned on a freezing microtome at $30 \mu \mathrm{m}$ thickness, and the sections were collected in PB. All brains were cut transversal to the main axis of the hindbrain.

Antibodies against SV2 (Buckley and Kelly, 1985) were used. Standard immunohistochemical procedures were followed using the avidin-biotin-peroxidase complex (ABC) method with reagents from Vectastain elite kits (Vector Laboratories, Burlingame, CA). For SV2 immunohistochemistry, sections were preincubated for $1 \mathrm{hr}$ in $0.5 \mathrm{M}$ Tris-HCl buffer, $\mathrm{pH} 7.6$, with $4 \%$ normal serum and $0.4 \%$ Triton $\mathrm{X}-100$ and then incubated overnight in SV2 supernatant (1:100-1:1000). Floating sections were incubated for $10 \mathrm{~min}$ in $3 \% \mathrm{H}_{2} \mathrm{O}_{2}$ in Tris- $\mathrm{HCl} /$ butanol (1:1), washed, incubated for $1 \mathrm{hr}$ in biotinylated secondary antibody diluted at
1:1000, washed, incubated in $\mathrm{ABC}$ for $1 \mathrm{hr}$, washed, incubated in biotinylated secondary antibody for $1 \mathrm{hr}$, washed, incubated in $\mathrm{ABC}$ for $1 \mathrm{hr}$, and then washed for $20 \mathrm{~min}$ in Tris- $\mathrm{HCl}$ followed by $20 \mathrm{~min}$ in actateimidazole buffer. Sections were treated with diaminobenzidine tetrahydrochloride $(0.48 \mathrm{mg} / \mathrm{ml})$ and $0.03 \% \mathrm{H}_{2} \mathrm{O}_{2}$ with nickel sulfate intensification $(26.3 \mathrm{mg} / \mathrm{ml})$ in acetate imidazole buffer. Sections were mounted onto subbed slides, dehydrated, cleared, and coverslipped with Permount. Some cases were additionally counterstained with neutral red.

Analysis. A blue/black reaction product characterized immunoreactive profiles. Analyses were performed on sections cut in the transverse plane. In the auditory hindbrain, the tonotopic axes in the NM and the NL proceed from rostromedial high best frequency to caudolateral low best frequency. Thus, sections cut in the transverse plane exhibit higher best frequency cells in a more medial position and lower best frequency cells in a more lateral position. Sections were photographed using a Magnafire camera attached to a Nikon microscope and processed with Adobe PhotoShop v.5.5 software to produce the final figures.

\section{Electron microscopy}

Five owl embryos (E17-E22) were processed for electron microscopy. Owls were anesthetized with ketamine $(33 \mathrm{mg} / \mathrm{kg}$, i.m.) followed by an overdose of Nembutal (100 mg/kg, i.v.) and perfused transcardially with either normal saline or avian Tyrodes solution containing (in $\mathrm{mM}$ ): 139 $\mathrm{NaCl}, 17 \mathrm{NaHCO}_{3}, 3 \mathrm{KCl}, 1 \mathrm{MgCl}, 3 \mathrm{CaCl}$, and 12.2 glucose, $\mathrm{pH} 7.3$, followed immediately by $2.5 \% \mathrm{EM}$ grade glutaraldehyde, $2 \%$ paraformaldehyde in $0.1 \mathrm{M}$ cacodylate buffer at $\mathrm{pH}$ 7.2. Embryos were cooled and perfused with saline followed by $1.5 \%$ paraformaldehyde followed by cold $2 \%$ paraformaldehyde $/ 2.5 \%$ glutaraldehyde. The brains were postfixed overnight at $4^{\circ} \mathrm{C}$. The brainstem was sectioned on a vibratome, and sections were washed in buffer and postfixed for $1 \mathrm{hr}$ at $4^{\circ} \mathrm{C}$ in a $1.0 \%$ solution of osmium tetroxide containing $7 \%$ dextrose. After postfixation, the tissue was again washed in several changes (10 min each) of buffer, dehydrated through a graded ethanol series, passed through two $15 \mathrm{~min}$ changes of propylene oxide, and infiltrated and embedded in Araldite resin. Thick sections $(0.35-1.0 \mu \mathrm{m})$ were mounted on glass slides and stained with toluidine blue. Ultrathin sections (pale gold-silver) taken from areas immediately adjacent to the thick sections were placed on formvar-coated wide slot grids and stained with 5.0\% uranyl acetate for $15 \mathrm{~min}$ and triple lead stain for $7 \mathrm{~min}$ at room temperature. 


\section{Rostral}
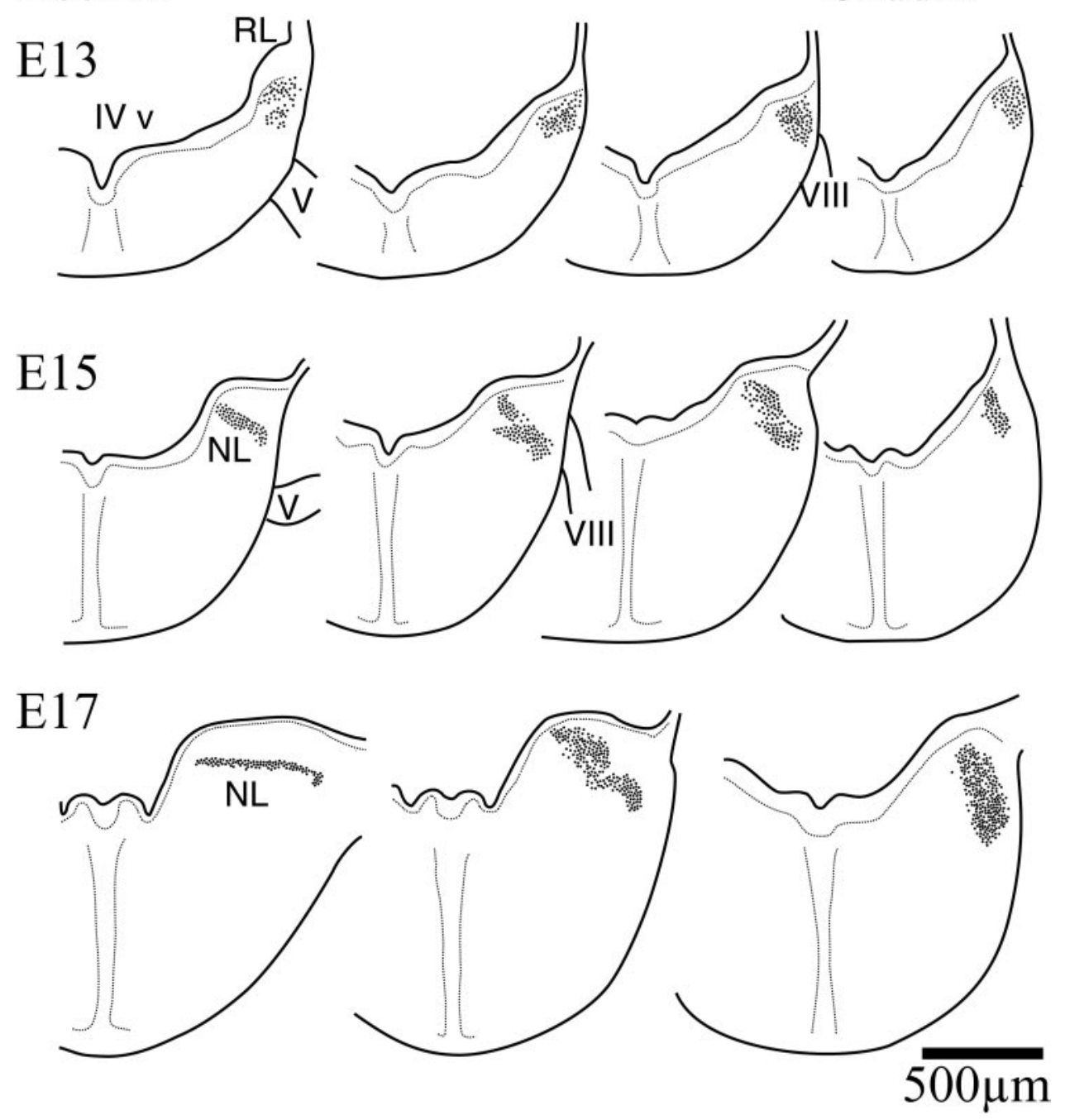

Figure 2. Schematic drawings of right hemisections through the hindbrain illustrating the early morphogenesis of the auditory hindbrain (dorsal toward the top). At E13, a columnar group of cells in the alar plate are located beneath the floor of the fourth ventricle near the rhombic lip. The separation of the nuclei begins to be evident at E15 toward the more rostral end of the column. At this age NL can be recognized in the more rostral regions of the column, and some separation of cell groups has begun in more caudal regions. By E17, the more rostral regions of the column have already differentiated into NL and assumed the typical laminar organization that is characteristic of basal birds. Separation of the cell groups continued in more caudal regions, but the most caudal regions of the column continued to exhibit the compact form. $I V v$, Fourth ventricle; $V$, trigeminal nerve; VIII, cochleovestibular nerve; $N L$, nucleus laminaris. For Figures 2 and 3, sections were taken from the rostral and caudal ends of the nuclei. Intermediate sections were chosen to illustrate differences in the organization of the cell groups.

\section{Quantification of synapses at the EM level}

Using adult material as a guide, excitatory synapses were characterized by asymmetric membrane densities associated with clusters of spherical vesicles (Carr and Boudreau, 1996). Symmetric membrane densities with oval or pleomorphic vesicles characterized inhibitory synapses. Synapses were quantified at the EM level in NL at different developmental stages of the owl (E17, E18, E19, E22, and E24; only E18, E22, and E24 are illustrated). Photomicrographs were taken of parallel transects through the rostral region of NL from dorsal to ventral at $10,000 \times$ magnification. Both dorsal and ventral ends of each transect were marked by a characteristic glial border (Cheng, 2001). Synapse number and location were then quantified at a final magnification of $25,000 \times$ on photographic prints. Synapses were identified by the presence of clear presynaptic and postsynaptic membrane densities and at least one associated vesicle. These criteria were used to eliminate the ambiguity between synapses and adhesion plaques, which also contained membrane densities and were common in embryonic tissue. The distribution of synapses was then determined by plotting the number of synapses as a function of their distance from the midline of NL. The dorsoventral distributions of synapses were aligned by their mediolateral position and plotted on a three-dimensional graph (see Fig. 7). A dip test of unimodality was used to describe synapse distribution (Hartigan and Hartigan, 1985; Sommer and McNamara, 1987). The dip statistic was calculated along with a $p$ value for rejecting the null hypothesis that the distribution is unimodal.

\section{RESULTS}

The auditory nerve of birds projects to two distinct cochlear nuclei: nucleus angularis (NA) and NM, homologous to the mammalian cochlear nucleus. The closely associated NL receives ipsilateral and contralateral inputs from NM and is where interaural time differences are first computed. The development of these three auditory nuclei is closely related.

\section{Primary morphogenesis}

The early morphogenesis of the cochlear nuclei and NL in the owl followed a sequence of events very similar to that described in 
chickens (Fig. 1) (Harkmark, 1954; Knowlton, 1967; Rubel et al., 1976; Book and Morest, 1990; Carr et al., 1998). A compact columnar mass of cells could be seen in the E13 owl embryo just below the fourth ventricle (IV v) and adjacent to the rhombic lip (Fig. 2, top). Throughout this initial morphogenetic period, this mass was surrounded by a clear neuropil that distinctively delineated this region of the alar plate (data not shown). This mass of cells constituted the anlagen of the cochlear nuclei and NL and, like the chicken, was located in the immediate vicinity of the neuroepithelium (Harkmark, 1954; Book and Morest, 1990). The mass extended rostrally to the level of the entry of the trigeminal nerve (V) and caudally to the entry of the vestibulocochlear nerve (VIII). NL became first identifiable at the most rostral extent of the column by E15 (Fig. 2, middle row, left). The separation of the auditory nuclei proceeded from rostral to caudal. By E17 the rostral end of NL could be identified and had acquired its typical embryonic laminar structure, whereas in the caudal hindbrain the anlagen remained a densely packed cluster of cells, and the auditory nuclei could not be differentiated (Fig. 2, bottom row).

The overall appearance of the owl rhombencephalon during this developmental period was remarkably similar to that of the chicken (Cramer et al., 2000a). In the chicken, the migration of NA neurons is slightly delayed with respect to NM and NL (Ariëns Kappers et al., 1967; Book and Morest, 1990). It appears that this is also the case in the owl, because NA was the last nucleus to be readily identifiable. Although the cochlear and laminaris nuclei began to separate between E15 and E17 in the rostral regions, the three immature nuclei could not be readily identified throughout their rostrocaudal extent until E18-19 (Fig. 3 , top row). The similarity between owl and chicken was not restricted to the development of the auditory areas. For example, the migratory streams identified by Harkmark (1954) in the chicken, which constituted the cells originating from the rhombic lip and presumably contributed in part to the inferior olive, were also evident in the owl material of equivalent phases of development (data not shown). The development of the hindbrain during this initial period of morphogenesis shared the sequences described for the chicken, albeit with longer time courses.

\section{Secondary morphogenesis}

During this second phase, NL neurons migrated apart from the compact laminar structure to assume the adult pattern near the time of hatching. Thus, the acquisition of the apomorphic pattern could be traced to this specific period of development, when the owl organization diverged from the plesiomorphic organization of basal birds (Sibley and Ahlquist, 1990). The distribution of cell bodies within the limits of NL changed during this secondary morphogenetic phase (Fig. 3). Owl neuronal somata in NL were initially restricted to the central region of the nucleus. The cell bodies were tightly packed, forming a compact lamina of somata surrounded dorsally and ventrally by a neuropil formed primarily by neuronal dendrites and NM axons (see below) (Fig. 3, E19, center). This neuropil clearly demarcated the external limits of NL. The redistribution of cells within the NL limits proceeded over time and along a rostromedial to caudolateral axis. A time series may be observed by comparing the most rostral E17 section (Fig. 2) with the E19 and caudal E23 sections (Fig. 3). At E19 the more rostromedial regions of the nucleus began to exhibit a redistribution of the neuronal somata (Fig. 3, compare E23 rostral section with caudal section). When the cells began to lose their bitufted morphology (see below), the somata of NL cells began to separate and to occupy the entire dorsoventral extension of the
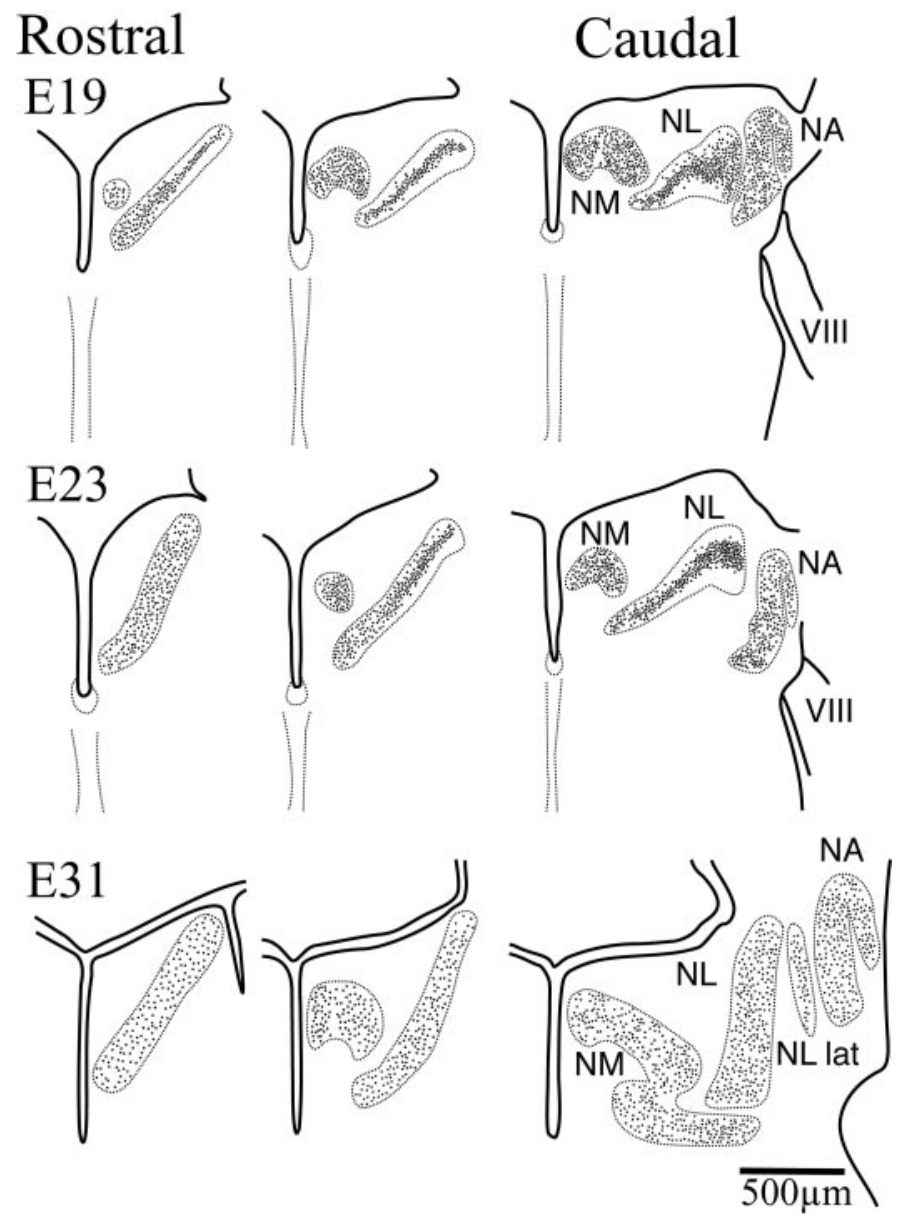

Figure 3. Schematic drawings of right hemisections through the hindbrain illustrating secondary morphogenesis in NL (dorsal toward the top). By E19, NM, NA, and NL were readily identified, although their limits still lay in close proximity in more caudal sections. The cells of the more caudolateral, low best frequency regions of $\mathrm{NL}$ were organized in a lamina typical of basal birds. In more rostromedial regions, however, cells in NL had begun to migrate away from the center of the lamina and fill the NL neuropil. This separation progressed toward more caudolateral regions such that by E24 this separation was evident in more caudal sections. By E31, close to hatching, the distribution of cells within NL was comparable to that seen in the adult, with cells occupying the entire nucleus all the way to the edges, although the nucleus had not yet reached its adult size. During the first 3 weeks after hatch, growth of NL was characterized by an increase in thickness that results in further separation of the NL cells. VIII, Cochleovestibular nerve; $R L$, rhombic lip; $N A$, nucleus angularis; $N M$, nucleus magnocellularis; $N L$, nucleus laminaris; $N L$ lat, lateral region of the nucleus laminaris.

nucleus. During these initial stages, the more caudolateral regions of the nucleus still exhibited the laminar structure that is characteristic of the plesiomorphic pattern (Fig. 3, E23, right). While development progressed, this separation of NL somata moved toward more caudolateral regions, such that by close to hatching the nucleus resembled the organization described for the adult owl (Fig. 3, E31). The most caudolateral, lower-frequency coding region of NL was an exception to this trend because it retained a monolayer-like structure and contained some bitufted neurons even in adulthood (Fig. 3, E31, right, NL lat) (Köppl and Carr, 1997).

The development of the elements of the ITD circuit in owls was similar to that of the chicken until fairly late in embryogenesis. In both species, and when dendrites began to form in $\mathrm{NL}$ 

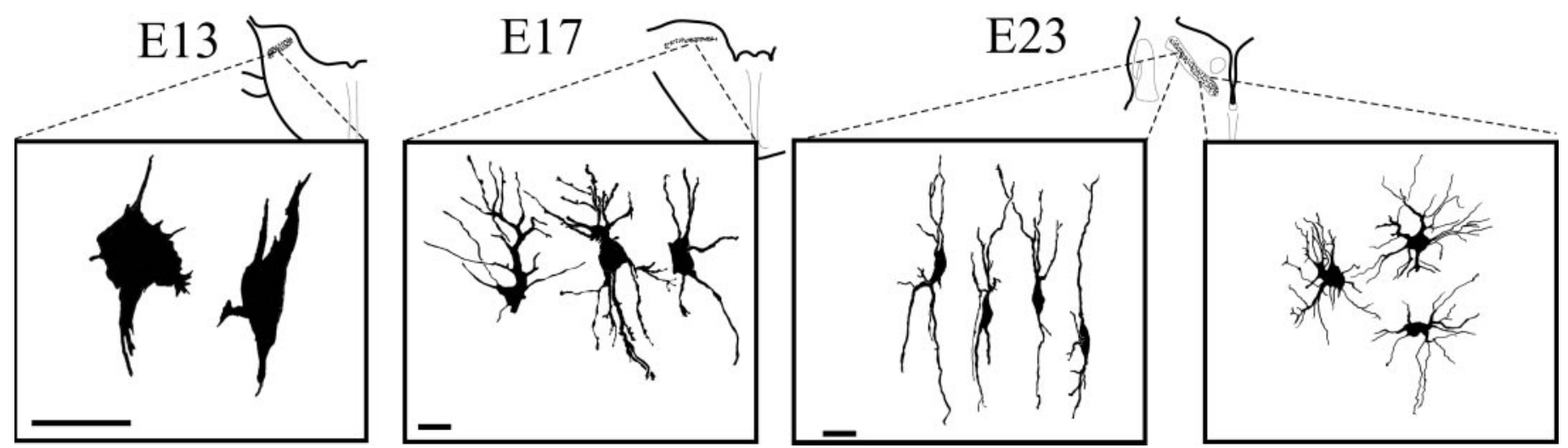

Figure 4. Morphology of cells in NL during secondary morphogenesis. At E13, Golgi material revealed the presence of neuroblast-like cells in the rostral ends of the cochlear anlage. By E17, when NL exhibited a laminar structure, NL cells began to exhibit dendritic polarization, with most of their dendrites projecting within the dorsoventral axis. By E23 in central NL, when the separation of the NL cells away from the lamina had begun, two distinct cell morphologies were observed. In the regions where the cells are confined to the middle of NL in a laminar structure, NL cells were clearly polarized with two dendritic tufts projecting dorsally and ventrally (left panel). In contrast, and on the same section, where NL has undergone the loss of the laminar organization, NL cells show dendrites distributed around their cell bodies and no obvious polarization (right panel). The morphology of the bitufted cells was reminiscent of the shape of the cells in NL in chickens, emus, and crocodilians, whereas the morphology of the cells after the loss of the laminar organization resembled that of the adult owl. NL cells continue to mature, and E31 neurons exhibit many short, fine dendrites (Carr and Boudreau, 1996). Scale bar, $20 \mathrm{~mm}$.
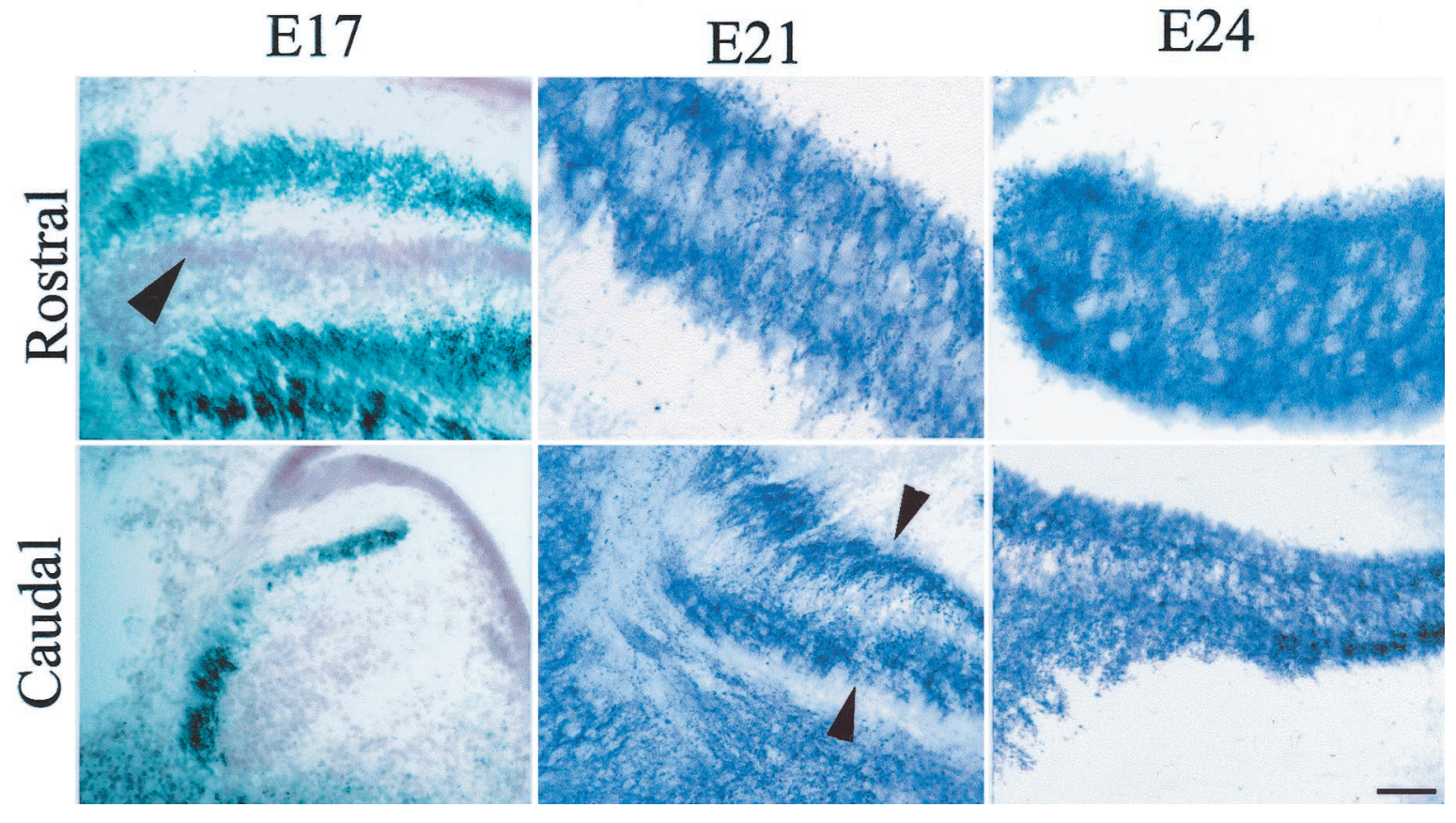

Figure 5. SV2 immunoreactive profiles at different ages of the owl embryo appear as dark puncta in photomicrographs of rostral and caudal sections through NL. At E17, in rostromedial regions, NL cells were arranged in the typical embryonic laminar structure (note neutral red-stained cell bodies, arrowhead), and darkly stained SV2 profiles were confined to the external edges of the nucleus. In the caudolateral region, the nuclei (NM and NL) have not become fully differentiated, and SV2 profiles were seen on the outside of the column. Cell bodies in the mass were labeled with neutral red (compare Fig. 2, E17, Caudal). By E21, NL has begun to undergo secondary morphogenesis at its more rostromedial extent, and SV2 profiles have migrated from their location on the distal dendrites into the neuropil of NL. In more caudal areas in the same embryo, the laminar structure is still compact, and SV2 profiles remain concentrated mainly toward the outside edges of the nucleus, in the distal dendrites (arrowheads). By E24, neurons in the rostral regions of NL show a distribution similar to that observed in the adult and lack the dendritic polarization that characterized them at earlier ages. SV2 profiles are rather evenly distributed throughout the dorsoventral dimension of the nucleus. More caudolateral regions of the nucleus at this age continue to exhibit more immature morphology, and SV2 profiles are denser in the outside edges (top, dorsal; left, lateral).

cells, NL appeared as a compact layer of bitufted cells that extended their dendrites in the dorsoventral axis (Fig. 4). Golgi material (Fig. 4) and immunocytochemical labeling with MAP-2 (data not shown) in E17 and E23 owl embryos showed NL neurons with the typical bitufted morphology that characterizes the homologous cells in chickens, emus, kiwis, and crocodiles
(Craige, 1930; Ariëns Kappers et al., 1967; Jhaveri and Morest, 1982; Soares et al., 1999, 2001; Kubke et al., 2002) (Fig. 4). These bitufted neurons were found in a compact layer in NL. In a rostromedial to caudolateral wave of development, the owl's NL neurons lost both their bitufted dendritic morphology and the compact layer organization typical of the plesiomorphic pattern 
(Figs. 3, 4). Starting in rostromedial NL at E19, the neurons retracted their thick dendrites and began a period of secondary dendritic growth during which they produced numerous fine dendrites, exhibiting a stellate appearance at first (Fig. 4, E23) (medial NL). This transition is illustrated in a drawing of a single Golgi section (Fig. 4, E23), where medial NL neurons have retracted their dendrites and separated from the compact layer, whereas their more lateral E23 counterparts still exhibit the younger phenotype. By E31, all except extreme low best frequency NL neurons had many short unpolarized dendrites (data not shown) (Carr and Boudreau, 1996). Typically, the dendrites were evenly distributed over the soma surface and did not show any evident dorsoventral polarity. Thus the change in dendritic morphology was a process that began in rostral NL at E19 and progressed in a caudally directed wave over the next $\sim 10 \mathrm{~d}$ of development.

Neurons in NL of the adult owl receive synapses from ipsilateral and contralateral NM around their entire cell bodies (Carr and Konishi, 1990). Early during development when the cells exhibited their typical bitufted morphology, the organization of NM terminals resembled that seen in the chicken. We used two techniques to visualize NM terminals: an antibody against synaptic vesicle protein SV2, which labels both synapses and growth cones, and ultrastructural localization of synapses. SV2 profiles were first observed as dark puncta at E17, primarily on the distal tip of the dendrites of the morphologically immature NL neurons (Fig. 5, E17 Rostral, E21 Caudal). When NL neurons reorganized their dendritic trees and began to separate from the original compact layer organization, SV2 profiles were found progressively closer to the NL somata (Fig. 5, E21 Rostral). The redistribution of synapses followed a rostromedial to caudolateral gradient, after the separation of NL cells away from the laminar array. Thus, at E17, in more rostral regions where NL has begun to differentiate, SV2 profiles were seen on the outside edges of the nucleus, presumably making synapses on the distal tips of NL dendrites. In more caudal regions, where NM and NL still form a mass, SV2-labeled profiles could be seen on the outside of the packed cell group (Fig. 5, E17 Caudal). By E21, in rostral sections, SV2 profiles had begun to invade the thickness of NL, whereas $\mathrm{SV} 2$ profiles were restricted to the outer edges of the nucleus in the more caudolateral regions of NL (Fig. 5, E21 Caudal). By E24, SV2 profiles were distributed fairly homogeneously within NL in the pattern typical of the adult owl (Fig. 5, E24).

Our interpretation of the data at the light level was supported by ultrastructural analysis. Figure 6 shows electron micrographs obtained from owl embryos at E18. In the region of NL where cells are arranged in a laminar structure, the neuronal somata were tightly packed with limited extracellular space. At higher magnification in an adjacent section (Fig. 6, inset), neuronal terminals formed synapses on dendritic structures within NL $(d$ in inset). These terminals were presumed to originate from NM, because it was the only excitatory input that NL neurons receive in the adult. These embryonic terminations exhibited typical synaptic densities and round synaptic vesicles characteristic of excitatory synapses (Carr and Boudreau, 1996). Thus, at least some of the SV2 immunoreactive terminals correspond to mature synaptic structures.

Because we could not differentiate between synaptic structures and immature nerve terminals with the SV2 antibody, we quantified the distribution of synapses along the dorsoventral axis of NL from EM material (Fig. 7). We constructed transects through rostral NL (compare with rostral sections in Fig. 3) and counted

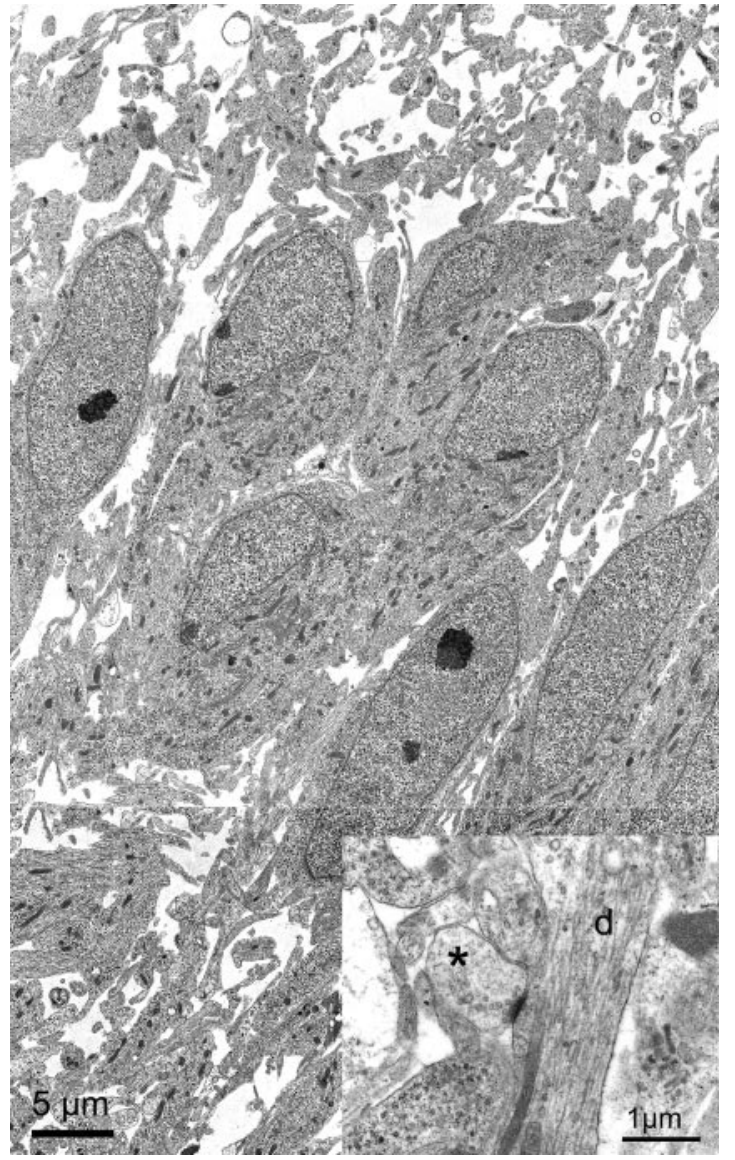

Figure 6. Electron micrographs obtained from NL at E18. Low-power electron micrographs of NL showed the neurons to be tightly packed in the center of the nucleus. In this region of NL, neurons exhibited polarized dendrites that project primarily in the dorsoventral dimension. Dendrites can be readily identified at larger magnification (inset, $d$ ). A synapse (asterisk) can be clearly identified making contact with an NL dendrite. The synapse is from an adjacent section.

numbers of synapses. Before the secondary morphogenetic phase, synapses were found primarily on the outer edges of the NL nucleus (Fig. 7, E18 lateral transects), which was devoid of neuronal somata and mainly contained dendritic processes. A dip test for unimodality confirmed that the distributions of synapses in these lateral transects were not unimodal (Fig. 7, legend). When the neurons separated to form the typical owl-like structure, the distribution of synapses became more evenly distributed throughout the dorsoventral axis of NL, and NM terminals were found distributed more evenly along the whole NL (Fig. 7, E22 medial transects, E24 transects).

Three secondary morphogenetic changes occurred during overlapping periods of time, making it difficult to establish causal relationships. These were the redistribution of cell bodies, dendritic retraction, and changes in synapse location. The secondary change in NL neuron morphology resulted from the retraction of the dendritic tufts and coincided spatially and temporally with the redistribution of cells away from the laminar assemblage, giving rise to the characteristic owl-like expanded structure. This also coincided with a redistribution of synapses across the NL boundaries and within the NL cells. Whether the synapses that have already formed on the NL dendrite are "pulled in" as the dendrites retract, or whether these original contacts are lost and new synapses are then established, cannot be determined at this time. 

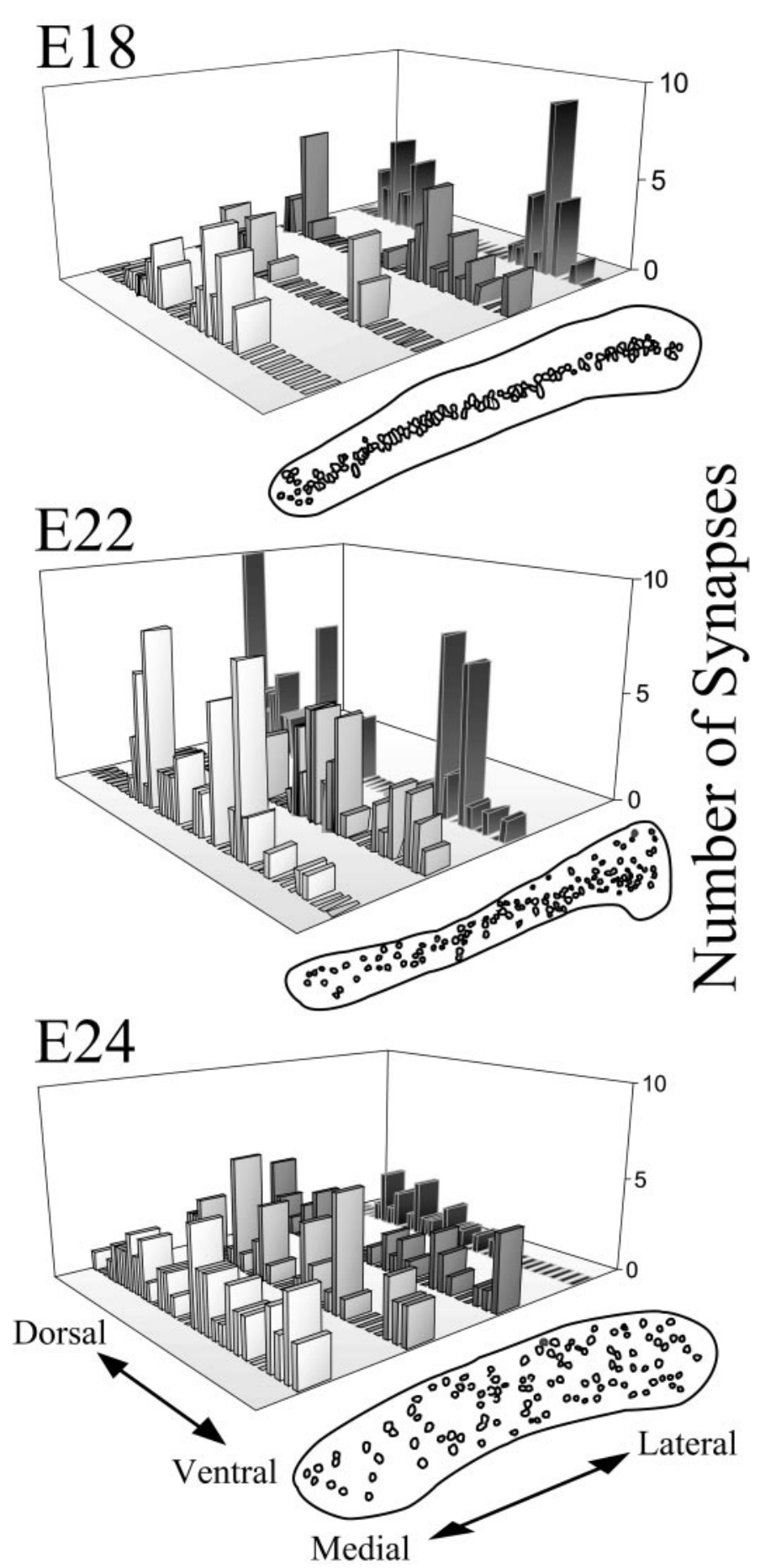

Figure 7. Quantification of synapse distribution along the dorsoventral dimension at three stages of development in NL (E18, E22, E24). Synapses were counted on transects across NL at the EM level. Camera lucida drawings correspond to the level of NL from which EM transects were obtained and show the location of the individual cell bodies in the adjacent semithick section. At E18, where NL continued to exhibit the laminar organization typical of the embryonic form, synapses were not found in the central regions of the nucleus. This was reflected in a dip test of each distribution. From medial to lateral, dip statistic and $p$ values for rejecting the hypothesis that the distribution is unimodal were as follows: dip statistic $=0.07, p=0.557 ; 0.11, p=0.006 ; 0.12, p=0.001 ; 0.14, p \leq$ 0.001 . Thus the distributions of synapses in all but the most medial transect were not unimodal. When cells began to migrate away from the laminar structure (E22), synapses became identifiable toward the center of the nucleus (E22, medial two transects; dip statistic $=0.07, p=0.014$

\section{DISCUSSION}

Owls are capable of great accuracy in detecting ITDs, and their auditory system shows morphological adaptations that contribute to this increased acuity. Among these are the asymmetry of the external ears, the organization of the facial ruff, an attenuation of tympanic interactions via the interaural canal, and hyperplasia of the auditory nuclei (Payne, 1971; Moiseff and Konishi, 1981; Calford and Piddington, 1988; Massoglia, 1997) (M. F. Kubke, C. E. Carr, unpublished observations).

The organization of NL, and its inputs from NM, varies considerably among different species of birds (Ariëns Kappers et al., 1967; Kubke and Carr, 2000). Basal birds, such as the emu and kiwi, as well as crocodilians, show an organization resembling that of the chicken (Craige, 1930; Carr and Code, 2000; Soares et al., 2001) (Kubke and Carr, unpublished observations). We assume that this organization represents a primitive (plesiomorphic) condition. The owl's organization must consequently be considered a derived (apomorphic) state. Given that ontogenetic changes must underlie circuit remodeling, comparisons between homologous circuits may identify which elements have been changed during evolution. Because the development of the plesiomorphic chicken auditory system has been studied thoroughly (for review, see Rubel and Parks, 1988; Kubke and Carr, 2000; Rubel and Fritzsch, 2002), we were able to compare the morphology of embryonic auditory structures in the owl with those of chicken. Because the morphological specializations seen in the auditory hindbrain of owls must result from changes to the ancestral pattern, we investigated the events that led to the reorganization of the owl map of ITDs during development.

Embryonic owl development closely resembled chicken development until late in embryogenesis, when the organization of NL took on the characteristic apomorphic form associated with the reorganization of the map of ITDs (Harkmark, 1954; Knowlton, 1967; Rubel et al., 1976; Book and Morest, 1990). The striking similarities between the early development of the chicken and owl auditory system support the hypothesis that small changes in the development of the NL circuit lead to the reorganization seen in owl NL and also to the behavioral changes in acuity observed in the owl (Nishikawa, 1997). The emergence of this novel secondary morphogenetic phase suggests that the reorganization of NL may be an adaptation for accurate detection of ITDs. This reorganization is characterized by three major events. These are the loss of the laminar organization, the retraction of dendrites in NL neurons, and the redistribution of NM-NL synapses.

The loss of the laminar organization found in basal birds and crocodilians appears to be a fundamental difference between chickens and owls and results in an expansion in the dorsoventral dimension. The laminar organization of NL in the chicken restricts the configuration in which ITD can be mapped to a single dimension along the mediolateral anatomical axis within each isofrequency band (Young and Rubel, 1983; Overholt et al., 1992; Joseph and Hyson, 1993). In contrast, the reorganization of the $\mathrm{NM}-\mathrm{NL}$ circuit in the owl gives rise to additional NM axonal

$\leftarrow$

for the medial and $0.05, p=0.422$ for the central transect). At this age, synapses were not found in the tightly packed center of the nucleus in more lateral regions $(E 22$, lateral transect; dip statistic $=0.111, p=$ $0.035)$. By $E 24$, NL had lost its laminar organization, and synapses were found throughout the entire dorsoventral extent of NL (all transects). From medial to lateral, dip statistic and $p$ values were as follows: dip = $0.059, p=0.187 ; 0.079, p=0.027 ; 0.11, p=0.002 ; 0.07, p=0.442$. 
segments within a newly added dorsoventral dimension. These segments provide the delay required for mapping the ITDs associated with the contralateral hemifield (Sullivan and Konishi, 1986; Carr and Boudreau, 1991). The short NM axonal segments within NL can provide the required delay within the spatial limits of the dorsoventral span of the nucleus because of a reduction of the internodal distance and axonal diameter, which results in slower conduction velocity within NL. Delayed myelination of these axonal segments has been proposed as a mechanism by which internodal distances are reduced (Carr, 1994; Cheng, 2001).

The segregation of ipsilateral and contralateral inputs onto dorsal and ventral dendrites appears to be an important feature in the ability of the neuron to perform coincidence detection in the chicken (Overholt et al., 1992; Agmon-Snir et al., 1998). This segregation, however, does not favor ITD computations at frequencies above $2 \mathrm{kHz}$ (Agmon-Snir et al., 1998; Simon et al., 1999). Owls accurately compute ITDs between 4 and $8 \mathrm{kHz}$, a range above that of other vertebrates (Konishi, 1999), and their NL neurons are characterized by many short unpolarized dendrites (Carr and Boudreau, 1993). The owl-like organization of $\mathrm{NL}$ can also be found in other bird species with hearing ranges that extend above $4 \mathrm{kHz}$ (Kubke, Dent, and Carr, unpublished observations). The loss of the laminar structure and the reorganization of the delay lines are accompanied by the loss of the bitufted NL cell morphology. Whether the redistribution of dendrites and synapses improves ITD computation or whether it is a consequence of external signals remains to be established.

The role of biochemical signaling has been shown in the NL in the chicken. Here, NM projects to the dorsal dendrites of ipsilateral NL neurons and to the ventral dendrites of contralateral NL neurons. Tyrosine kinase signaling may be involved in establishing these spatially segregated connections (Cochran et al., 1999; Cramer et al., 2000b). When NM-NL projections are forming, EphA4 expression in NL is asymmetric, with higher expression in the dorsal NL neuropil than in the ventral neuropil. At the same time, a complementary pattern of tyrosine kinase B receptor is observed with higher levels of expression in the ventral neuropil. The interplay between these two signaling systems may serve to guide growing axons to the appropriate region (Cochran et al., 1999; Cramer et al., 2000b). Developmental studies in owl may reveal whether these expression patterns have changed.

Improvements in behavioral acuity can be the result of modifications of a single circuit or increases in the areas devoted to a given computation. Examples of the latter include increasing the number and expanse of visual areas in primates and auditory areas in bats (Suga et al., 1987; Northcutt and Kaas, 1995; Krubitzer et al., 1997). These increases in cortex most likely result from prolonged development and protracted neurogenesis (Finlay and Darlington, 1995). Circuit modification occurs during development of the ITD circuit in the brainstem of the owl. Secondary morphogenetic events remodel the NM-NL circuit and its associated azimuthal map. This circuit modification may contribute to the increased acuity of sound localization in owls. The nervous system is evolutionarily conservative, and small changes in structure can lead to profound changes in function and behavior (Nishikawa, 1997). Understanding how these morphogenetic changes take place in the context of increased behavioral function should illuminate mechanisms by which neural circuits evolve.

\section{REFERENCES}

Agmon-Snir H, Carr C, Rinzel J (1998) The role of dendrites in auditory coincidence detection. Nature 393:268-272.

Ariëns Kappers CU, Huber GC, Crosby EC (1967) The comparative anatomy of the nervous system of vertebrates, including man. New York: Hafner.

Book KJ, Morest DK (1990) Migration of neuroblasts by perikaryal translocation: role of cellular elongation and axonal outgrowth in the acoustic nuclei of the chick embryo medulla. J Comp Neurol 297:55-76.

Buckley K, Kelly RB (1985) Identification of a transmembrane glycoprotein specific for secretory vesicles of neuronal and endocrine cells. J Cell Biol 100:1284-1294.

Calford MB, Piddington RW (1988) Avian interaural canal enhances interaural delay. J Comp Physiol [A] 162:503-510.

Carr C, Code R (2000) The central auditory system in reptiles and birds. In: Comparative hearing: birds and reptiles (Dooling R, Fay R, Popper A, eds). New York: Springer.

Carr CE (1994) The development of nucleus laminaris in the barn owl. In: 10th international symposium on hearing (Manley GA, Klump GM, Koppl C, Fastl H, Oekinghaus H, eds), pp 24-31. Singapore: World Scientific Publishers.

Carr CE, Boudreau RE (1991) Central projections of auditory nerve fibers in the barn owl. J Comp Neurol 314:306-318.

Carr CE, Boudreau RE (1993) Organization of the nucleus magnocellularis and the nucleus laminaris in the barn owl: encoding and measuring interaural time differences. J Comp Neurol 334:337-355.

Carr CE, Boudreau RE (1996) Development of the time coding pathways in the auditory brain stem of the barn owl. J Comp Neurol 373:467-483.

Carr CE, Konishi M (1988) Axonal delay lines for time measurement in the owl's brainstem. Proc Natl Acad Sci USA 85:8311-8315.

Carr CE, Konishi M (1990) A circuit for detection of interaural time differences in the brainstem of the barn owl. J Neurosci 10:3227-3246.

Carr CE, Kubke MF, Massoglia DP, Cheng SM, Rigby L, Moiseff A (1998) Development of temporal coding circuits in the barn owl. In: Psychophysical and physiological advances in hearing (Palmer AR, Rees A, Summerfield AQ, Meddis R, eds), pp 344-351. London: Whurr.

Cheng S (2001) Glial influences on the development of auditory delay lines in barn owls. PhD thesis, University of Maryland.

Cochran S, Stone J, Bermingham-McDonogh O, Akers S, Lefcort F, Rubel E' (1999) Ontogenetic expression of trk neurotrophin receptors in the chick auditory system. J Comp Neurol 413:271-288.

Craige EH (1930) Studies on the brain of the kiwi (Apteryx australis). J Comp Neurol 49:223-357.

Cramer K, Fraser S, Rubel E (2000a) Embryonic origins of auditory brain-stem nuclei in the chick hindbrain. Dev Biol 224:138-151.

Cramer K, Rosenberger M, Frost D, Cochran S, Pasquale E, Rubel E (2000b) Developmental regulation of EphA4 expression in the chick auditory brainstem. J Comp Neurol 426:270-278.

Finlay BK, Darlington RB (1995) Linked regularities in the development and evolution of mammalian brains. Science 268:1578-1584.

Harkmark W (1954) Cell migrations from the rhombic lip to the inferior olive, the nucleus raphe and the pons. A morphological and experimental investigation on chick embryos. J Comp Neurol 100:115-209.

Hartigan JA, Hartigan PM (1985) The dip test of unimodality. Ann Statistics 13:70-84.

Jeffress L (1948) A place theory of sound localization. J Comp Physiol Psychol 41:35-39.

Jhaveri S, Morest D (1982) Neuronal architecture in nucleus magnocellularis of the chicken with observations on nucleus laminaris: a light and electron microscope study. Neuroscience 7:809-836.

Joseph AW, Hyson RL (1993) Coincidence detection by binaural neurons in the chick brain stem. J Neurophysiol 69:1197-1211.

Knowlton VY (1967) Correlation of the development of membranous and bony labyrinths, acoustic ganglia, nerves, and brain centers of the chick embryo. J Morphol 121:179-208.

Konishi M (1999) Deciphering the brain's codes. In: Neural codes and distributed representations: foundations of neural computation (Abbott L, Sejnowski TJ, eds), pp 1-18. Cambridge, MA: MIT.

Köppl C, Carr CE (1997) Low-frequency pathway in the barn owl's auditory brainstem. J Comp Neurol 378:265-282.

Krubitzer K, Kunzle H, Kaas J (1997) Organization of sensory cortex in a Madagascan insectivore, the Tenrec (Echinops telfairi). J Comp Neurol 379:399-414.

Kubke MF, Carr CE (1998) Development of AMPA-selective glutamate receptors in the auditory brainstem of the barn owl. Microsc Res Tech 41:176-186.

Kubke MF, Carr CE (2000) Development of the auditory brainstem of birds: comparison between barn owls and chickens. Hear Res 147:1-20.

Kubke MF, Gauger B, Basu L, Wagner H, Carr C (1999) Development of calretinin immunoreactivity in the brainstem auditory nuclei of the barn owl (Tyto alba). J Comp Neurol 415:189-203. 
Kubke MF, Carr CE, Dooling RJ (2002) Organization of nucleus laminaris in different species of birds. Assoc Res Otolaryngol Abstr 426.

Massoglia D (1997) Embryonic development of the time coding nuclei in the brainstem of the barn owl (Tyto alba). MS thesis, University of Maryland.

Meyer S, Wagner H (1995) Guidance of retinal axons by membrane components derived from the barn owl's optic tectum. Zoology 99:103-112.

Moiseff A, Konishi M (1981) The owl's interaural pathway is not involved in sound localization. J Comp Physiol 144:299-304.

Moiseff A, Konishi M (1983) Binaural characteristics of units in the owl's brainstem auditory pathway: precursors of restricted receptive fields. J Neurosci 3:2553-2562.

Nishikawa K (1997) Emergence of novel functions during brain evolution. Bioscience 47:341-354.

Northcutt R, Kaas J (1995) The emergence and evolution of mammalian neocortex. Trends Neurosci 18:373-379.

Overholt EM, Rubel EW, Hyson RL (1992) A circuit for coding interaural time differences in the chick brainstem. J Neurosci 12:1698-1708.

Payne RS (1971) Acoustic localization of prey by barn owls (Tyto alba). J Exp Biol 54:535-573.

Ramón y Cajal S (1908) Les ganlions terminaux du nerf acoustique des oiseaux. Trab Inst Cajal Invest Biol 6:195-225.

Rubel EW, Fritzsch B (2002) Auditory system development: primary auditory neurons and their targets. Annu Rev Neurosci 25:51-101.

Rubel EW, Parks TN (1988) Organization and development of the avian brain-stem auditory system. In: Brain function (Edelman GM, Einar Gall W, Maxwell Cowan W, eds), pp 3-92. New York: Wiley.

Rubel EW, Smith DJ, Miller LC (1976) Organization and development of brain stem auditory nuclei of the chicken: ontogeny of n. magnocellularis and n. laminaris. J Comp Neurol 166:469-490.
Sibley CG, Ahlquist JE (1990) Phylogeny and classification of birds: a study in molecular evolution. New Haven, CT: Yale UP.

Simon J, Carr CE, Shamma S (1999) A dendritic model of coincidence detection in the avian brainstem. Neurocomputing 26:263-269.

Smith Z (1981) Organization and development of brain stem auditory nuclei of the chicken: dendritic development in n. laminaris. J Comp Neurol 203:309-333.

Smith ZDJ, Rubel EW (1979) Organization and development of brain stem auditory nuclei of the chicken: dendritic gradients in nucleus laminaris. J Comp Neurol 186:213-239.

Soares D, Simon J, Carr CE (1999) The cochlear nuclei of the caiman. Soc Neurosci Abstr 24:249.9.

Soares D, MacLeod K, Carr CE, Simon JZ (2001) The substrate for ITD detection in the Emu (Dromaius novaehollandiae). Soc Neurosci Abstr 27:747.7.

Sommer CJ, McNamara JN (1987) Power considerations for the dip test of unimodality using mixtures of normal and uniform distributions. Am Stat Assoc: Proc Stat Comput Sect 186-191.

Stensaas L (1967) The development of hippocampal and dorsolateral pallial regions of the cerebral hemisphere in fetal rabbits. 3. Twentynine millimeter stage, marginal lamina. J Comp Neurol 130:149-162.

Suga N, Niwa H, Taniguchi I, Margoliash D (1987) The personalized auditory cortex of the mustached bat: adaptation for echolocation. J Neurophysiol 58:643-654.

Sullivan WE, Konishi M (1986) Neural map of interaural phase difference in the owl's brainstem. Proc Natl Acad Sci USA 83:8400-8404.

Valverde F (1970) The Golgi method. A tool for comparative structura analysis. In: Contemporary research methods in neuroanatomy (Nauta WJH, Ebbesson SOE, eds), pp 12-28. New York: Springer.

Young SR, Rubel EW (1983) Frequency-specific projections of individual neurons in chick brainstem auditory nuclei. J Neurosci 3:1373-1378. 